\title{
A study of VLF wave propagation characteristics in the earth-ionosphere waveguide
}

\author{
M. Indira Devi ${ }^{1}$, I. Khan ${ }^{2}$, and D. N. Madhusudhana Rao ${ }^{1}$ \\ ${ }^{1}$ Department of Physics, Andhra University, Visakhapatnam 530 003, A. P. India \\ ${ }^{2}$ Department of Instrumentation Technology, College of Engineering, Andhra University, Visakhapatnam 530 003, A. P. India
}

(Received January 11, 2008; Revised April 2, 2008; Accepted April 9, 2008; Online published August 4, 2008)

In the light of newly found applications for very low frequency (VLF) (3-30 kHz) measurements in the prediction of earthquakes and the detection of lightning discharges and gamma ray bursts, there has been a revival of interest in the study of VLF propagation in the earth-ionosphere waveguide. The propagation characteristics of VLF radiowaves in the earth-ionosphere waveguide critically depend upon the lower ionospheric ionization, which determines the conductivity profile of the upper boundary of the waveguide. In this context, it is potentially worthwhile to revisit the waveguide mode analysis to compute propagation parameters of long-distance VLF transmissions while taking into account different approximations to the ionospheric conductivity. We have carried out a waveguide mode analysis of $16-\mathrm{kHz}$ VLF waves traveling great distances, assuming three different models for conductivity. The computational results show that at heights of less than $70 \mathrm{~km}$, the change in the phase of the VLF waves due to changes in phase velocity is smaller when the ionosphere is sharply bound and assumed to have finite conductivity rather than infinite conductivity. The effect of a non-sharp or diffuse boundary at the top of the earth-ionosphere waveguide is found to cause a lowering of the height of reflection.

Key words: VLF propagation, earth-ionosphere waveguide.

\section{Introduction}

Very low frequency (VLF) radiowaves in the frequency range $3-30 \mathrm{kHz}$ have long been in use for long-distance communication and navigation. As these waves exhibit high-phase stability, they have found application in standard frequency and time information dissemination. In addition, VLF phase and amplitude measurements have facilitated the study of the $D$-region of the ionosphere, since the height of the reflection of these waves lies entirely in the $D$-region both day and night, and they have also been used for solar flare patrolling. Although recent years have seen the increased use of satellite-based communication systems and navigational (GPS) aids, ground-based systems are still useful because they are seen as reliable alternatives to the satellite systems, which are vulnerable to hazards in space and the vagaries of space weather. Submarine communication still relies on VLF transmitters. There has also been a renewed interest in VLF in the light of some recent studies showing seismic activity signatures on VLF records. These findings suggest that such measurements may facilitate earthquake prediction (Singh et al., 2005; Chakrabarti et al., 2007). In addition, there have been reports of perturbations induced in VLF transmissions by lightning discharges, $\gamma$-ray bursts and meteor activity (Inan et al., 1996; Cummer, 2000; Otsuyama et al., 2003; De et al., 2006). In this context, model studies of propagation characteristics of VLF waves are critically important and are being carried

Copyright (c) The Society of Geomagnetism and Earth, Planetary and Space Sciences (SGEPSS); The Seismological Society of Japan; The Volcanological Society of Japan; The Geodetic Society of Japan; The Japanese Society for Planetary Sciences; TERRAPUB. out by many workers (Cummer, 2000; Grubor et al., 2005).

The propagation of VLF waves over long distances is generally treated as propagation through a waveguide constituted by the earth-considered a perfect conductor-as one wall and the lower ionosphere ( $D$-region) as the other. An important problem in VLF communication is the variation in propagation time due to change in the velocity of propagation caused by variation in the width of earthionosphere waveguide. The $D$-region ionization, being solar controlled, undergoes regular and irregular variations. These changes in ionization alter the height of reflection of the VLF signals and thereby the width of the earthionosphere waveguide; consequently, the velocity of propagation is modified. This is clearly evident at night, when the height of the reflection of the VLF waves increases by about $15-20 \mathrm{~km}$ from its daytime value, resulting in a considerable increase in the separation between the walls of the waveguide. As a consequence of this separation, the phase velocity of the waves decreases and the signals will be delayed at the receiver. The phase velocity, however, is determined by the nature of the conductivity profile of the ionosphere. Therefore, it is important to have an understanding of the propagation characteristics in the waveguide to be able to interpret the experimental results as well as to use these waves for earthquake predictions.

We report here our study of the propagation of $16-\mathrm{kHz}$ VLF waves to great distances through the earth-ionosphere waveguide. This frequency is chosen since phase variation measurements of $16-\mathrm{kHz}$ transmissions originating from Rugby $\left(52.3^{\circ} \mathrm{N}, 1.2^{\circ} \mathrm{W}\right)$, UK, and made at Visakhapatnam $\left(17.7^{\circ} \mathrm{N}, 83.3^{\circ} \mathrm{E}\right)$, India, are available. 


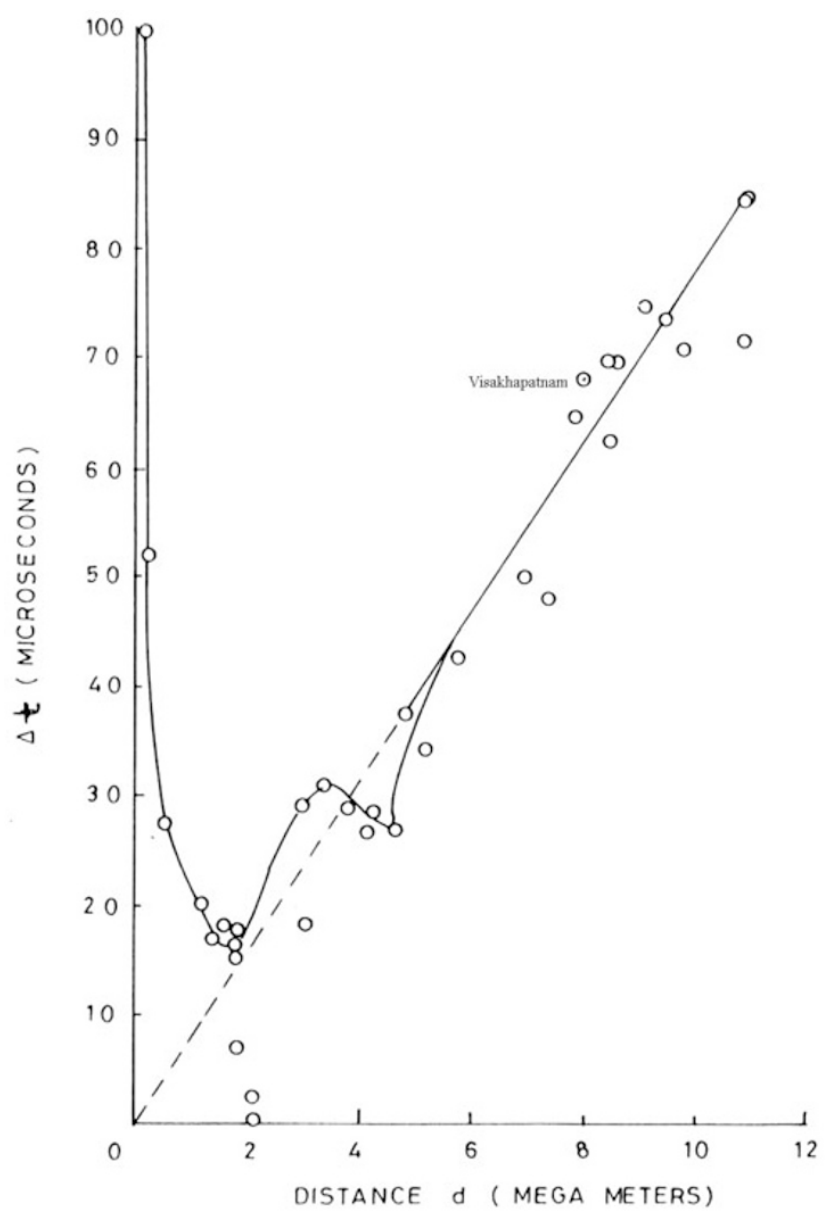

Fig. 1. Variation in diurnal change of transmission time with distance (after Chilton et al., 1964).

\section{Model Computations}

Model calculations of wave propagation in the earthionsophere waveguide generally employ the waveguide mode theory (Wait, 1970). Other methods, such as direct finite-difference time-domain (FDTD) modeling, are also being used for such calculations (Cummer, 2000 and references therein). However, in any of these methods the upper boundary of the waveguide, namely the ionosphere, is treated with different approximations in terms of its conductivity. In a comparative study of mode theory and FDTD method, Cummer (2000) observed that, in general, there is an excellent agreement between the computational results obtained using these two methods. Here, in the present study, the computations are based on the mode theory.

Conventional model computations consider a waveguide whose lower boundary is a perfectly conducting earth and whose upper boundary is a sharply bounded ionosphere with either a finite conductivity or infinite conductivity or a non-sharp (diffuse) ionosphere (Kikuchi, 1986; Reznikov et al., 1993). Here, we also the propagation characteristics of 16-kHz transmissions through the Rugby-Visakhapatnam path that have been computed using the waveguide mode equations for the three approximations to the conductivity profile.
Table 1. Change in phase of the $16-\mathrm{kHz}$ signal for a transition from the all-day to all-night path for different daytime reflection heights, assuming an infinitely conducting ionosphere.

\begin{tabular}{cccc}
\hline S. no. & $h(\mathrm{~km})$ & $\frac{v_{1 \mathrm{~d}}}{c}$ & $\frac{\Delta \varphi}{\Delta h} \mathrm{deg} / \mathrm{km}$ \\
\hline 1 & 60 & 0.99834 & 27.85 \\
2 & 65 & 0.99749 & 24.55 \\
3 & 70 & 0.99674 & 22.10 \\
4 & 75 & 0.99606 & 20.30 \\
5 & 80 & 0.99543 & 18.90 \\
6 & 85 & 0.99484 & 17.80 \\
7 & 90 & 0.99428 & 16.90 \\
8 & 95 & 0.99375 & 16.25 \\
9 & 100 & 0.99324 & 15.70 \\
\hline
\end{tabular}

\subsection{Sharply bounded ionosphere with infinite and fi- nite conductivity}

Wait (1959) has shown that the phase velocity of the $n$th order mode of radiowaves passing through the earth and a sharply bounded ionosphere, assuming the earth and the ionosphere to possess infinite conductivity, is approximately given by

$$
v_{n}=c\left(1-C_{n}^{2}\right)^{-\frac{1}{2}}\left(1-\frac{h}{2 a}\right)
$$

where $c$ is the velocity of light in free space $\left(3 \times 10^{8} \mathrm{~m} / \mathrm{s}\right)$, $C_{n}=\left(n-\frac{1}{2}\right) \frac{\lambda}{2 h}, h$ is the height of reflection of the VLF wave mode from the ionosphere and $a$ and $\lambda$ are the earth's radius and wavelength of the wave (corresponding to 16$\mathrm{kHz}$ Rugby transmissions in the present case), respectively. At great distances (greater than $5000 \mathrm{~km}$ ) from the transmitter, such as in the case reported here, where the great circle distance between Rugby and Visakhapatnam is $8023 \mathrm{~km}$, the attenuation of higher order modes will be very high. Hence, only the first order $(n=1)$ mode needs to be considered. As such, Eq. (1) becomes

$$
v_{1}=c\left(1-\frac{\lambda^{2}}{16 h^{2}}\right)^{-\frac{1}{2}}\left(1-\frac{h}{2 a}\right) .
$$

If $v_{1 \mathrm{~d}}$ and $v_{1 \mathrm{n}}$ are the phase velocities of the $16-\mathrm{kHz}$ transmissions for the all-day and all-night paths, respectively, the change in phase velocity will be

$$
\frac{\Delta v_{1}}{c}=\frac{v_{1 \mathrm{n}}-v_{1 \mathrm{~d}}}{c} \cong \Delta\left\{\left(1-\frac{h}{2 a}\right)\left(1-\frac{\lambda^{2}}{16 h^{2}}\right)^{-\frac{1}{2}}\right\},
$$

which can be approximated to

$$
\begin{aligned}
\frac{\Delta v_{1}}{c} & \cong \Delta\left\{\left(1-\frac{h}{2 a}\right)\left(1+\frac{\lambda^{2}}{32 h^{2}}+\cdots\right)\right\} \\
& \cong-\left(\frac{h}{2 a}+\frac{\lambda^{2}}{16 h^{2}}\right) \frac{\Delta h}{h} .
\end{aligned}
$$

It can be easily shown from Eqs. (3) and (4) that the difference in time of travel from the all-day path to the all-night path, $\Delta t_{1}$, becomes

$$
\frac{\Delta t_{1}}{d}=\left(\frac{c}{v_{1 \mathrm{n}}}-\frac{c}{v_{1 \mathrm{~d}}}\right) \times \frac{10^{3}}{0.3} \quad \mu \mathrm{s} / \mathrm{Mm}
$$


Table 2. Change in phase of the $16-\mathrm{kHz}$ signal for a transition from the all-day to all-night path for different daytime reflection heights, assuming a finitely conducting ionosphere.

\begin{tabular}{ccccc}
\hline S. no. & $h(\mathrm{~km})$ & $10^{3} C_{1 \mathrm{~d}}^{2}$ & $\frac{v_{1 \mathrm{~d}}}{c}$ & $\frac{\Delta \varphi}{\Delta h} \mathrm{deg} / \mathrm{km}$ \\
\hline 1 & 60 & $(-0.365+i 1.436)$ & 1.000183 & 24.3 \\
2 & 65 & $(-1.185+i 1.380)$ & 0.999407 & 23.4 \\
3 & 70 & $(-2.679+i 1.333)$ & 0.998660 & 22.7 \\
4 & 75 & $(-4.127+i 1.295)$ & 0.997936 & 22.1 \\
5 & 80 & $(-5.536+i 1.262)$ & 0.997232 & 21.5 \\
6 & 85 & $(-6.919+i 1.234)$ & 0.996540 & 21.1 \\
7 & 90 & $(-8.262+i 1.210)$ & 0.995869 & 20.7 \\
8 & 95 & $(-9.586+i 1.189)$ & 0.995207 & 20.4 \\
9 & 100 & $(-10.889+i 1.170)$ & 0.994555 & 20.1 \\
\hline
\end{tabular}

where $d$ is the distance of travel. Based on the phase variation measurements of the $16-\mathrm{kHz}$ transmissions from Rugby made at Visakhapatnam over a period of nearly 2 years, the average diurnal change in transmission time $(\Delta t)$ is $8.5 \mu \mathrm{s} / \mathrm{Mm}$ (Khan et al., 2001). This value compares very well with earlier measurements, as can be seen from Fig. 1, in which this value of $\Delta t$ is indicated on the plot of $\Delta t$ versus distance given by Chilton et al. (1964) for several propagation path lengths. It can also be inferred from this figure that modal interference is important up to about $5.5 \mathrm{Mm}$ and that beyond this distance, only the first order mode becomes significant and $\Delta t$ varies linearly with distance with a slope of $7.75 \mu \mathrm{s} / \mathrm{Mm}$. Since the changes in time and phase $(\Delta \varphi)$ are related through

$$
\Delta \varphi=\Delta t 360 f \quad \text { degrees }
$$

with $f$ being the frequency, the change in phase for a 1$\mathrm{km}$ change in the day-to-night height of reflection can be shown, using Eqs. (4), (5) and (6), as

$$
\begin{aligned}
& \frac{\Delta \varphi}{\Delta h}= 5.76 \times 10^{6}\left(\frac{1}{2 a}+\frac{\lambda^{2}}{16 h^{3}}\right) \\
& \cdot \frac{d}{c}\left(\frac{c}{v_{1 \mathrm{~d}}}\right)^{2} \text { degrees } / \mathrm{km} .
\end{aligned}
$$

The ratio of the daytime phase velocity of the first order mode to free space velocity $\left(v_{1 \mathrm{~d}} / c\right)$ and $\Delta \varphi / \Delta h$ have been calculated using Eqs. (2) and (7), with $\lambda=18.75 \mathrm{~km}$, which corresponds to the $16-\mathrm{kHz}$ Rugby transmission frequency, and $d=8023 \mathrm{~km}$ for the Rugby-Visakhapatnam path, for different heights of reflection. The results are presented in Table 1.

Wait (1963) has studied the characteristics of VLF waves propagating to great distances through a waveguide constituted by the conducting earth and sharply bounded ionosphere with finite conductivity and showed that the first order mode phase velocity is given by

$$
\frac{v_{1}}{c}=1+\operatorname{Re}\left(\frac{C_{1}^{2}}{2}\right)
$$

in which $C_{1}^{2}$ is given by

$$
C_{1}^{2}=\frac{\frac{7 \pi}{6}-\frac{2 k a}{3}\left(\frac{2 h}{a}\right)^{\frac{3}{2}}-i \alpha\left(\frac{2 h}{a}\right)^{\frac{1}{2}}}{k a\left(\frac{2 h}{a}\right)^{\frac{1}{2}}+\frac{i \alpha}{2}\left(\frac{2 h}{a}\right)^{-\frac{1}{2}}} .
$$

In Eq. (9), apart from the symbols already defined, $k=2 \pi / \lambda$, the propagation constant, and $\alpha=$ $-2 i^{1 / 2}\left(\omega / \omega_{\mathrm{r}}\right)^{1 / 2}\left(1-i \omega_{\mathrm{r}} / \omega\right)$, where $\omega$ is the angular frequency of the VLF wave and $\omega_{\mathrm{r}}$ is the ionospheric conductivity parameter given by $\omega_{\mathrm{r}}=\omega_{\mathrm{o}}^{2} / v, \omega_{\mathrm{o}}$, with $v$ being the angular plasma frequency and the effective collision frequency, respectively. $\alpha$ is a parameter that defines the ionospheric reflection coefficient $R$ through

$$
R=-\exp \left(\alpha C_{1}^{1}\right)
$$

where $C_{1}^{1}$ is the cosine of the angle of incidence given by

$$
C_{1}^{1}=\left(C_{1}^{2}+\frac{2 h}{\alpha}\right)^{\frac{1}{2}}
$$

for the first order mode.

The change in phase for a $1-\mathrm{km}$ change in reflection height, $\Delta \varphi / \Delta h$, and the ratio of daytime phase velocity to free space velocity $\left(v_{1 \mathrm{~d}} / c\right)$ for different all-day path reflection heights ranging from 60 to $100 \mathrm{~km}$ in steps of $5 \mathrm{~km}$ have been calculated using Eqs. (7) and (8) with a finite value for conductivity, $\omega_{\mathrm{r}}=2 \times 10^{5}$. The results are presented in Table 2.

A comparison of results presented in Tables 1 and 2 shows that $v_{1 \mathrm{~d}} / c$ is larger for the case of a sharply bounded ionosphere with finite conductivity than for the case of infinite conductivity - i.e. phase velocities are larger for the finite conductivity case. Further, it can be seen that for reflection heights 60 and $65 \mathrm{~km}, \Delta \varphi / \Delta h$ is smaller in the case of the finitely conducting than infinitely conducting sharply bounded ionosphere, whereas it is larger at and above $70 \mathrm{~km}$. Also, the spread in $\Delta \varphi / \Delta h$ is only $4.2^{\circ} / \mathrm{km}$ as $h$ increases from 60 to $100 \mathrm{~km}$ for the finite conductivity model, whereas in the case of the infinite conductivity model, it is as much as $12.15^{\circ} / \mathrm{km}$.

\subsection{Diffuse ionosphere with exponentially varying con- ductivity}

A non-sharp or diffuse ionosphere is considered here, in which the conductivity of the $D$-region changes exponentially (Wait, 1963) as

$$
\omega_{\mathrm{r}}=\omega_{\mathrm{ro}} \exp [\beta(z-h)]
$$

where $\omega_{\text {ro }}$ is the value of $\omega_{\mathrm{r}}$ at the reference height, $z=h$, taken as $2.5 \times 10^{5}$ following Wait (1963) and $\beta$ is the height gradient. The reflection coefficient for such an exponential 
Table 3. Cosine of the complex angle of incidence, $C_{1}^{1}$ as a function of altitude for the first order mode.

\begin{tabular}{|c|c|c|c|c|c|c|c|c|c|}
\hline S. no. & $h(\mathrm{~km})$ & $\frac{2 h}{a} \times 10^{3}$ & $\left(C_{\mathrm{r}}^{2}-C_{\mathrm{i}}^{2}\right) 10^{3}$ & $\left(2 C_{1 \mathrm{i}} C_{1 \mathrm{r}}\right) 10^{3}$ & $\left(4 C_{1 \mathrm{i}}^{2} C_{1 \mathrm{r}}^{2}\right) 10^{3}$ & $\left(p_{1 \mathrm{r}}^{2}-p_{1 \mathrm{i}}^{2}\right)^{*}$ & $\left(p_{1 \mathrm{r}}^{2}+p_{1 \mathrm{i}}^{2}\right)^{\#}$ & $p_{1 \mathrm{r}}$ & $p_{1 \mathrm{i}}$ \\
\hline 1 & 60 & 18.847 & 0.365 & 1.436 & 2.062 & 19.212 & 19.266 & 0.1387 & 0.0052 \\
\hline 2 & 65 & 20.418 & -1.185 & 1.380 & 1.904 & 19.233 & 19.282 & 0.1388 & 0.0049 \\
\hline 3 & 70 & 21.988 & -2.679 & 1.333 & 1.777 & 19.309 & 19.355 & 0.1390 & 0.0048 \\
\hline 4 & 75 & 23.559 & -4.127 & 1.295 & 1.6777 & 19.432 & 19.475 & 0.1395 & 0.0046 \\
\hline 5 & 80 & 25.130 & -5.536 & 1.262 & 1.593 & 19.594 & 19.635 & 0.1401 & 0.0045 \\
\hline 6 & 85 & 26.700 & -6.919 & 1.234 & 1.523 & 19.781 & 19.819 & 0.1407 & 0.0044 \\
\hline 7 & 90 & 28.271 & -8.262 & 1.210 & 1.464 & 20.009 & 20.046 & 0.1415 & 0.0043 \\
\hline 8 & 95 & 29.841 & -9.586 & 1.189 & 1.414 & 20.255 & 20.290 & 0.1424 & 0.0042 \\
\hline 9 & 100 & 31.412 & -10.889 & 1.170 & 1.369 & 20.523 & 20.556 & 0.1433 & 0.0041 \\
\hline
\end{tabular}

Table 4. $\left(\alpha_{\mathrm{r}}+i \alpha_{\mathrm{i}}\right)$ for a diffuse ionosphere for different values of $\beta$.

\begin{tabular}{cccc}
\hline \multirow{2}{*}{ S. no. } & Height $(\mathrm{km})$ & $\beta=0.5$ per km & $\beta=0.3$ per km \\
\cline { 3 - 4 } & $h$ (reference height) & $-3.126+i 1.333$ & $-3.126+i 1.333$ \\
2 & $h-1$ & $-2.888+i 0.585$ & $-2.961+i 0.877$ \\
3 & $h-2$ & $-2.891-i 0.126$ & $-2.862+i 0.441$ \\
4 & $h-3$ & $-2.952-i 0.846$ & $-2.828+i 0.015$ \\
5 & $h-4$ & $-3.258-i 1.618$ & $-2.858+i 0.411$ \\
6 & $h-5$ & $-3.769-i 2.492$ & $-2.952+i 0.846$ \\
\hline
\end{tabular}

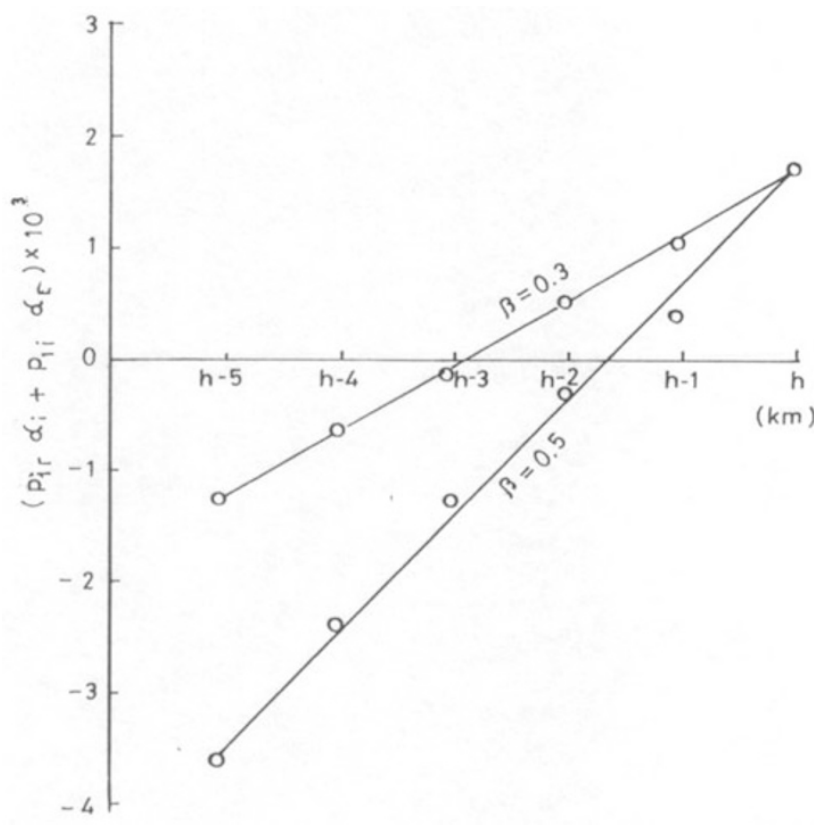

Fig. 2. Change in height of reflection for the diffusive parameters for $\beta=$ 0.5 and $0.3 \mathrm{~km}^{-1}$.

layer may also be expressed in the form $-\exp \left(\alpha C_{1}^{1}\right)$, as in Eq. (10), assuming $\alpha$ to be nearly constant for a wide range of angles of incidence. Writing $\alpha=\alpha_{\mathrm{r}}+i \alpha_{\mathrm{i}}$, the reflection coefficient of the first order mode of the $16-\mathrm{kHz}$ VLF signal may be written as

$$
\begin{aligned}
R & =-\exp \left(\alpha C_{1}^{1}\right)=\exp (-\pi) \exp \left(\alpha C_{1}^{1}\right) \\
& =\exp \left(p_{1 \mathrm{r}} \alpha_{\mathrm{r}}-p_{1 \mathrm{i}} \alpha_{\mathrm{i}}\right) \exp \left\{-i\left(\pi+p_{1 \mathrm{r}} \alpha_{\mathrm{i}}+p_{1 \mathrm{i}} \alpha_{\mathrm{r}}\right)\right\}
\end{aligned}
$$

In Eq. (13), $\exp \left(p_{1 \mathrm{r}} \alpha_{\mathrm{r}}-p_{1 \mathrm{i}} \alpha_{\mathrm{i}}\right)$ is the amplitude of the reflection coefficient and $-\left(\pi+p_{1 \mathrm{r}} \alpha_{\mathrm{i}}+p_{1 \mathrm{i}} \alpha_{\mathrm{r}}\right)$ is its phase; $p_{1 \mathrm{r}}$ and $p_{1 \mathrm{i}}$ are given by

$$
\begin{aligned}
p_{1 \mathrm{r}}+i p_{1 \mathrm{i}}= & C_{1}^{1}=\left(C_{1}^{2}+\frac{2 h}{a}\right)^{\frac{1}{2}} \\
& +\left(C_{1 \mathrm{r}}^{2}-C_{1 \mathrm{i}}^{2}+\frac{2 h}{a}+i 2 C_{1 \mathrm{r}} C_{1 \mathrm{i}}\right)^{\frac{1}{2}}
\end{aligned}
$$

with $\left(C_{1 \mathrm{r}}^{2}-C_{1 \mathrm{i}}^{2}\right)$ and $2 C_{1 \mathrm{r}} C_{1 \mathrm{i}}$ as the real and imaginary parts of $C_{1}^{2}$, respectively. The real and imaginary quantities $p_{1 \mathrm{r}}$ and $p_{1 \mathrm{i}}$ have been computed for different altitudes and are given in Table 3. It may be noted from this table that the real part of $C_{1}^{1}$ changes from 0.1387 to 0.1433 as the height changes from 60 to $100 \mathrm{~km}$ and that the imaginary part changes from $5.2 \times 10^{-3}$ to $4.1 \times 10^{-3}$ in the same height interval. The real and imaginary parts of the parameter defining the reflection coefficient, namely $\alpha_{\mathrm{r}}$ and $\alpha_{\mathrm{i}}$, have also been computed with $\omega_{\mathrm{r}}=2.5 \times 10^{5}$ and $\beta=0.5$ and $0.3 \mathrm{~km}^{-1}$, taking the reference height as $70 \mathrm{~km}$ and $z$ varying from 65 to $70 \mathrm{~km}$ in steps of $1 \mathrm{~km}$. The results are presented in Table 4. In order that the phase of the reflection coefficient of the first order mode be $-\pi$ for the nonisotropic or diffuse ionosphere with exponentially varying conductivity, the quantity $\left(p_{1 \mathrm{r}} \alpha_{\mathrm{i}}+p_{1 \mathrm{i}} \alpha_{\mathrm{r}}\right)$ must vanish. In Fig. 2, the value of this quantity is plotted as a function of $(h-\Delta h)$, with $\Delta h$ in steps of $1 \mathrm{~km}$. An examination of this figure shows that in the case of the diffuse ionosphere, the height of reflection is reduced by about $1.65 \mathrm{~km}$ for $\beta=0.5 \mathrm{~km}^{-1}$ and by about $2.8 \mathrm{~km}$ for $\beta=0.3 \mathrm{~km}^{-1}$, from the reference level $h$. The result obtained here-that the effect of the non-sharp boundary at the top of the con- 
centric earth-ionosphere waveguide-causes a depression in the height of reflection, is consistent with what has been reported by Wait (1963).

\section{Conclusion}

Waveguide mode analysis of VLF waves of a $16-\mathrm{kHz}$ frequency traveling a distance of more than $8 \mathrm{Mm}$ has been carried out to study their propagation parameters while taking into account three different conductivity models for the ionosphere, the upper boundary of the waveguide, with the conducting earth as the lower wall. The computational results show that below $70 \mathrm{~km}$ the change in phase due to changes in phase velocity is smaller when the ionosphere is assumed to have finite conductivity than when it is infinitely conducting. We have also found that the effect of a nonsharp or diffuse boundary at the top of the earth-ionosphere waveguide is to lower the height of reflection.

Acknowledgments. The authors wish to express their gratitude to Prof. K. V. V. Ramana for his interest and valuable suggestions while carrying out this work.

\section{References}

Chakrabarti, S., S. Sasmal, M. Saha, R. Khan, D. Bhoumik, and S. K. Chakrabarti, Unusual behavior of $D$-region ionization time at $18.2 \mathrm{kHz}$ during seismically active days, Indian J. Phys., 81, 531-538, 2007.

Chilton, C. J., D. D. Crobie, and A. G. Jean, Phase variations in VLF propagation, in Propagation of radiowaves at frequencies $300 \mathrm{kc} / \mathrm{s}$, edited by Blackband, 257-290, Pergamon Press, London, 1964.

Cummer, S. A., Modeling electromagnetic propagation in the earthionosphere waveguide, IEEE Trans. Antennas Propagat., 48, 1420-
1429, 2000.

De, S. S., D. K. De, A. Guha, and P. K. Mandal, Detection of 2004 Leonid meteor shower by observing its effects on VLF transmission, Indian Radio Space Phys., 35, 396-400, 2006.

Grubor, D., D. Sulic, and V. Zigman, Influence of solar X-ray flares on the earth-ionosphere waveguide, Serb. Astron. J., 171, 29-35, 2005.

Inan, U. S., A. Slingeland, V. P. Pasko, and J. V. Rodriguez, VLF and LF signatures of mesospheric and ionospheric response to lightning discharges, J. Geophys. Res., 101, 5219-5229, 1996.

Khan, I., Y. V. P. K. Raghava, and D. N. Madhusudhana Rao, Seasonal variation of diurnal shift in the height of reflection of $16 \mathrm{kHz}$ VLF signals recorded at Visakhapatnam, Proc. A. P. Akad. Sci., 5, 215-219, 2001.

Kikuchi, T., Waveguide model analyses of Omega VLF wave propagation at $13.6 \mathrm{kHz}$, J. Atmos. Terr. Phys., 48, 15-23, 1986.

Otsuyama, T., T. Kariya, and M. Hayakawa, VLF signatures of ionospheric perturbations associated with winter lightning in the Hokuriku area of Japan, Adv. Polar Upper Atmos. Res., 17, 109-119, 2003.

Reznikov, A. E., A. I. Sukhorukov, D. E. Edemskii, V. V. Kopeikin, P. A. Morozov, B. S. Ryabov, A. Yu. Shchekotov, and V. V. Solov'ev, Investigations of the lower ionosphere over Antarctica via ELF-VLF radiowaves, Antarctic Sci., 5, 107-113, 1993.

Singh, B., V. Kushwah, V. Singh, and M. Tomar, Simultaneous ULF/VLF amplitude anomalies observed during moderate earthquakes in Indian region, Indian Radio Space Phys., 34, 221-232, 2005.

Wait, J. R., Diurnal change of ionospheric heights deduced from phase velocity measurements at VLF, Proc. IRE, 47, 998, 1959.

Wait, J. R., Influence of lower ionosphere on propagation of VLF waves to great distances, J. Res. NBS, 67D, 375-381, 1963.

Wait, J. R., Electromagnetic waves in stratified media (2nd Edition), Pergmon Press, Oxford, 1970.

M. Indira Devi (e-mail: indiramalladi@yahoo.com), I. Khan, and D. N. Madhusudhana Rao 